\title{
Monitoring the ripening process of Cheddar cheese based on hydrophilic component profiling using gas chromatography-mass spectrometry
}

\author{
H. Ochi, ${ }^{*} \dagger$ Y. Sakai, ${ }^{*}$ H. Koishihara,‡ F. Abe, ${ }^{*}$ T. Bamba, $\dagger$ and E. Fukusaki ${ }^{1}$ \\ ${ }^{*}$ Food Science \& Technology Institute, Morinaga Milk Industry Co. Ltd., 5-1-83 Higashihara Zama, Kanagawa 252-8583, Japan \\ †Department of Biotechnology, Graduate School of Engineering Osaka University, 2-1 Yamadaoka, Suita, Osaka 565-0871, Japan \\ †Food Research \& Development Institute, Morinaga Milk Industry Co. Ltd., 5-1-83 Higashihara Zama, Kanagawa 252-8583, Japan
}

\section{ABSTRACT}

We proposed an application methodology that combines metabolic profiling with multiple appropriate multivariate analyses and verified it on the industrial scale of the ripening process of Cheddar cheese to make practical use of hydrophilic low-molecular-weight compound profiling using gas chromatography-mass spectrometry to design optimal conditions and quality monitoring of the cheese ripening process. Principal components analysis provided an overview of the effect of sodium chloride content and kind of lactic acid bacteria starter on the metabolic profile in the ripening process of Cheddar cheese and orthogonal partial least squares-discriminant analysis unveiled the difference in characteristic metabolites. When the sodium chloride contents were different (1.6 and $0.2 \%$ ) but the same lactic acid bacteria starter was used, the 2 cheeses were classified by orthogonal partial least squares-discriminant analysis from their metabolic profiles, but were not given perfect discrimination. Not much difference existed in the metabolic profile between the 2 cheeses. Compounds including lactose, galactose, lactic acid, 4-aminobutyric acid, and phosphate were identified as contents that differed between the 2 cheeses. On the other hand, in the case of the same salt content of $1.6 \%$, but different kinds of lactic acid bacteria starter, an excellent distinctive discrimination model was obtained, which showed that the difference of lactic acid bacteria starter caused an obvious difference in metabolic profiles. Compounds including lactic acid, lactose, urea, 4-aminobutyric acid, galactose, phosphate, proline, isoleucine, glycine, alanine, lysine, leucine, valine, and pyroglutamic acid were identified as contents that differed between the 2 cheeses. Then, a good sensory prediction model for "rich flavor," which was defined as "thick and rich, including umami taste and soy sauce-like flavor," was constructed based on the metabolic profile during

Received April 4, 2013.

Accepted August 19, 2013.

${ }^{1}$ Corresponding author: fukusaki@bio.eng.osaka-u.ac.jp ripening using partial least squares regression analysis. The amino acids proline, leucine, valine, isoleucine, pyroglutamic acid, alanine, glutamic acid, glycine, lysine, tyrosine, serine, phenylalanine, methionine, aspartic acid, and ornithine were extracted as ripening process markers. The present study is not limited to Cheddar cheese and can be applied to various maturation-type natural cheeses. This study provides the technical platform for designing optimal conditions and quality monitoring of the cheese ripening process.

Key words: metabolomics, metabolic profiling, cheese ripening, gas chromatography-mass spectrometry

\section{INTRODUCTION}

Natural cheese is made from cow milk by first adding rennet and lactic acid bacteria starter. The final cheese is obtained after a multi-step manufacturing process. Biochemical changes occur in the cheese, especially during ripening, resulting in the development of a specific flavor, aroma, and texture (McSweeney, 2004). Several factors, including the composition of the ingredients (added salts, enzymes, and lactic acid bacteria) and the degradation and catabolism that occur during ripening, result in considerable diversity in the compounds that lead to the phenotype expressed as cheese quality. Sensory characteristics of natural cheese are expressed in terms of synergistic nonlinear interactions between various components, making these characteristics difficult to elucidate and control scientifically by targeting a single or just a few components. In the cheese industry, it is difficult to scientifically grasp the complex components affecting the quality of cheese and the dynamic change during maturation in relation to the quality of cheese; thus, artisan skills based on long-term experience are still required to manage the quality and manufacturing.

A variety of metabolites are formed during the vital activity of microorganisms, plants, and animals. The whole metabolites are called metabolomes and the area of comprehensive analytical study of metabolomes is metabolomics. Because metabolomics can analyze 
many kinds of massive compounds uniformly and exhaustively, it helps to reveal the high resolution of phenotype and metabolic fluctuation. Metabolomics is an effective post-genomic research tool that has been applied to many disciplines, including the study of human diseases, nutrition, drug discovery, and plant physiology (Wishart, 2008; Drexler et al., 2011; Herrero et al., 2012; Ma et al., 2012; Nadella et al., 2012). Because metabolic profiling based on metabolomics is an extremely effective tool not only for the analysis of biological metabolisms, but also for the analysis of food science processes, the application of metabolic profiling for monitoring the quality, processing, and safety of both raw materials and final products in food science has recently attracted attention (Cevallos-Cevallos et al., 2009; Aiello et al., 2011; Cevallos-Cevallos and Reyes-De-Corcuera, 2012; Kuang et al., 2012; Mannina et al., 2012; Putri et al., 2013). Metabolomics holds major promise as an effective tool for solving the problem of qualitatively evaluating the complexity of cheese.

Recent studies have focused on the hydrophilic tasteactive compounds in cheese, such as the water-soluble components in Cheddar cheese (Andersen et al., 2010), Gouda cheese (Toelstede and Hofmann, 2008), Swiss cheese (Biede and Hammond, 1979a,b), Comte cheese (Salles et al., 1995), and goat milk cheese (Engel et al., 2000). Therefore, the application of metabolic profiling of the hydrophilic components in cheese represents a major advance toward scientifically expressing the sensory characteristics of cheese. It is meaningful if we can understand the sensory qualities of cheese in terms of the components of the metabolome and use this understanding to design optimal manufacturing conditions for quality.

Based on this idea, we conducted metabolic profiling using GC-MS, targeting hydrophilic low-molecularweight components in Cheddar and Gouda cheeses with various degrees of ripening, successfully resulting in the construction of highly precise sensory prediction models for 2 sensory attributes expressing important parts of maturation, "rich flavor" and "sour flavor" (Ochi et al., 2012b). Additionally, for using the metabolomicsbased cheese quality evaluation tool at manufacturing sites, we conducted metabolic fingerprinting using a gas chromatography-flame ionization detector and successfully reconstructed the sensory prediction model with metabolic profiling from GC-MS as previously reported (Ochi et al., 2012a). Our previous reports revealed that metabolomics-based component profiling, focusing on hydrophilic low-molecular-weight components, was able to predict the important aspects of the sensory characteristics related to ripening, identify the contributing compounds, and be expanded practically to manufacturing sites.
The results obtained from diverse cheese samples with various degrees of ripening could be applicable to cases in which a given kind of natural cheese changes its metabolome during the ripening process, resulting in changing sensory characteristics. However, no published research exists validating this. The focus of the present study is not so much to precisely describe the specific case of Cheddar cheese manufacturing in terms of the transition of component compounds as to demonstrate the methodology applicable to universal cases, which means the novel framework for monitoring cheese ripening, by linking metabolome analysis to multivariate analysis and validate its effectiveness.

In this study, we conducted metabolic profiling targeting hydrophilic low-molecular-weight compounds with GC-MS for cheese samples with different sodium chloride contents or kinds of lactic acid bacteria starter, changing over ripening time under different ripening temperatures. The objectives of this study were (1) to reveal the differences in metabolic profile caused by manufacturing conditions such as ingredients, (2) to construct a model corresponding to sensory characteristics using metabolic profiling, and (3) to investigate the compounds that may work as markers of cheese maturation.

\section{MATERIALS AND METHODS}

\section{Cheddar Cheese Samples}

Three kinds of Cheddar cheese were manufactured by Morinaga Milk Industry Co. Ltd. (Higashihara Zama, Kanagawa, Japan) in the usual way (Scott, 1998) and coded as A, B, and C. They were manufactured at the same production site using the same sort of raw milk. The differences in these samples were as follows: the same lactic acid bacteria starter was used for A and B, but A was a normal salt type and cheese B was a saltfree type. Cheese $\mathrm{C}$ was a normal salt type, whereas the lactic acid bacteria starter used was a different kind from that of cheese A and cheese B. Thirteen samples, consisting of 1 sample before ripening and 12 samples under different ripening temperatures and ripening periods, were collected for $\mathrm{A}, \mathrm{B}$, and $\mathrm{C}$. The ripening conditions were set as follows: the ripening temperatures were 5,10 , and $15^{\circ} \mathrm{C}$ and the ripening periods were 1,3 , 6 , and 9 mo. Ripening was conducted as a $20-\mathrm{kg}$ block of cheese. Collected samples of a predetermined period under each temperature condition were reserved at a superchilling temperature $\left(-3^{\circ} \mathrm{C}\right)$ to stop maturation until sensory evaluation.

The composition of cheeses A, B, and C (before ripening) was as follows: $34.8 \%$ moisture content, $26.5 \%$ protein content, $34.2 \%$ fat content, and $1.6 \%$ sodium 
chloride $(\mathrm{NaCl})$ content for cheese A; $36.58 \%$ moisture content, $27.9 \%$ protein content, $32.6 \%$ fat content, and $0.2 \% \mathrm{NaCl}$ content for cheese $\mathrm{B}$; and $33.7 \%$ moisture content, $27.2 \%$ protein content, $34.6 \%$ fat content, and $1.6 \% \mathrm{NaCl}$ content for cheese C.

\section{Reagents}

All chemicals used in this study were of analytical grade. Methanol and chloroform were used as extraction solvents, ribitol was used as an internal standard, and pyridine was used as a solvent (Wako Pure Chemical Industries Ltd., Osaka, Japan). Methoxyamine hydrochloride was purchased from Sigma (St. Louis, MO). $N$-Methyl- $N$-(trimethylsilyl) trifluoroacetamide was purchased from GL Sciences Inc. (Tokyo, Japan).

\section{Sample Preparation of Cheeses for GC-MS Analysis}

Cheese samples were frozen in liquid nitrogen, ground, and then freeze-dried. Freeze-dried cheese $(100 \mathrm{mg})$ in 2-mL Eppendorf tubes was extracted with $1,000 \mu \mathrm{L}$ of methanol: $\mathrm{H}_{2} \mathrm{O}: \mathrm{CHCl}_{3} \quad(2.5: 1: 1, \mathrm{vol} / \mathrm{vol} /$ vol). Sixty microliters of $0.2 \mathrm{mg} / \mathrm{mL}$ ribitol, used as an internal standard, was added to the mixture, mixed with 5-mm-diameter zirconia beads using a vortex mixer, suspended in a ball-mill $(20 \mathrm{~Hz} ; 1 \mathrm{~min}$ at room temperature), and then sonicated $(1.5 \mathrm{~min} \times 3)$. The sample was centrifuged at $16,000 \times g$ for 3 min at $4^{\circ} \mathrm{C}$, and then $800 \mu \mathrm{L}$ of the supernatant was transferred to a 1.5-mL Eppendorf tube. Water $(400 \mu \mathrm{L})$, purified using a Millipore Milli-Q system (Millipore Corp., Bedford, MA), was added and the sample was vortexed. Following centrifugation $\left(16,000 \times g\right.$ for $3 \mathrm{~min}$ at $\left.4^{\circ} \mathrm{C}\right), 500$ $\mu \mathrm{L}$ of supernatant was transferred to another $1.5-\mathrm{mL}$ Eppendorf tube and capped. The cap was subsequently pierced and the extract was evaporated to remove methanol in a centrifuge vacuum concentrator at room temperature for approximately $2 \mathrm{~h}$. After evaporation, the extract was freeze-dried in a glass bottle at room temperature overnight.

\section{Sample Derivatization}

For derivatization, $100 \mu \mathrm{L}$ of methoxyamine hydrochloride in pyridine $(20 \mathrm{mg} / \mathrm{mL})$ was added to the above freeze-dried sample and the mixture was incubated in a Thermomixer comfort (Eppendorf, Tokyo, Japan) at $30^{\circ} \mathrm{C}$ for $90 \mathrm{~min}$ to induce the methoxyation reaction. Fifty microliters of $\mathrm{N}$-methyl- $\mathrm{N}$-(trimethylsilyl) trifluoroacetamide, a second derivatizing agent, was added and the mixture was incubated at $37^{\circ} \mathrm{C}$ for $90 \mathrm{~min}$ to induce the silylation reaction. The samples were then used for GC-MS analysis.

\section{GC-MS Analysis}

The gas chromatograph coupled with a mass spectrometer used in this study was a GCMS-QP2010 Ultra system (Shimadzu Corp., Kyoto, Japan) equipped with a $30-\mathrm{m} \times 0.25-\mathrm{mm}$ i.d. fused silica capillary column coated with a $0.25-\mu \mathrm{m}$ film of CPSIL 8 CB (Agilent Technologies Inc., Santa Clara, CA), and an AOC-20is series injector (Shimadzu Corp.) autosampler. The injection temperature was $230^{\circ} \mathrm{C}$. The carrier gas (helium) flow rate through the column was $1 \mathrm{~mL} / \mathrm{min}$. The column temperature was held at $80^{\circ} \mathrm{C}$ for $2 \mathrm{~min}$ isothermally and then was raised $15^{\circ} \mathrm{C} / \mathrm{min}$ to $330^{\circ} \mathrm{C}$ and held for $6 \mathrm{~min}$ isothermally. The transfer line temperature was $250^{\circ} \mathrm{C}$ and the ion source temperature was $200^{\circ} \mathrm{C}$. Ions were generated at $70 \mathrm{eV}$ with electron ionization and were recorded at 10,000 scans per second over the mass range of $m / z 85$ to 500 , with the detector voltage at $900 \mathrm{~V}$.

\section{Data Processing and Peak Identification}

Raw data acquired from GC-MS were processed by GCMSsolution software (Shimadzu Corp.), in which automatic peak detection, mass spectrum smoothing, and baseline correction were performed, followed by conversion into an AIA file (ANDI files: Analytical Data Interchange protocol, *.cdf). The ANDI format allowed conversion and transfer of data between different mass spectrum data systems. Peak retention times were aligned and the peak intensities were normalized to the ribitol peak included as the internal standard using MetAlign software (Lommen, 2009). Output of the result was presented in a comma-separated values data format, followed by identification of the peaks using AIoutput software (Tsugawa et al., 2011). Significant compounds were identified by comparing their mass spectra and retention times with those in libraries [the National Institute of Standards and Technology library and an in-house library prepared from authentic standard chemicals].

\section{Descriptive Sensory Analysis of Cheese Samples}

Quantitative descriptive sensory analysis (QDA; Stone and Sidel, 2004) was performed to evaluate the cheese sample $\mathrm{C}$ using a 15 -cm horizontal line with directional terms (e.g., weak/strong) located $13 \mathrm{~mm}$ from each end. Evaluation was performed by a trained panel of 10 members between 30 and $40 \mathrm{yr}$ of age (4 women and 6 men). Individuals were selected based on their ability to detect 5 basic tastes (salty, sweet, acidic, bitter, and umami) and their ability to identify differences between the cheeses using the triangle test (Meilgaard 
et al., 2006). Using QDA methodology, terms for the evaluation of the cheese samples were developed by the panel. Quantitative descriptive sensory analysis was conducted for appearance, aroma, flavor, texture, and aftertaste. Ballot development and panelist calibration were accomplished during 6 working sessions, each lasting approximately $90 \mathrm{~min}$. Eight $1.5-\mathrm{cm} \times 1.5-\mathrm{cm}$ $\times 1.0-\mathrm{cm}$ cheese samples, at approximately $5^{\circ} \mathrm{C}$, were placed in a single $70-\mathrm{mL}$ plastic cup and served to a panelist. A balanced block (MacFie et al., 1989) order of samples and panelists was used to avoid an order effect and all samples were evaluated 3 times. Samples were identified by a random 3-digit number when presented to the panel. The entire evaluation was conducted in a sensory evaluation room that was air-conditioned and free of external aromas, noise, and distractions at the Research and Information Center of Morinaga Milk Industry Co. Ltd. (Zama City, Japan).

\section{Statistical Analysis}

Analysis of variance was carried out with JMP software (SAS Institute Inc., Cary, NC). Multivariate analysis of the results was performed. Principal components analysis (PCA), partial least squares (PLS) projections to latent structures, and orthogonal PLSdiscriminant analysis (OPLS-DA; Wiklund et al., 2008) were performed with SIMCA-P software (version 12.0; Umetrics AB, Umeå, Sweden) to analyze the descriptive and instrumental data.

Differences among cheese samples A, B, and C were analyzed by statistical discriminant analysis using OPLS-DA and compounds contributing to discrimination were extracted by S-plot. Orthogonal PLS is a modified method of the PLS that separates the systematic variation in $\mathrm{X}$ into 2 parts: one that is linearly related to $\mathrm{Y}$ (and therefore predictive) and one that is orthogonal to Y. The OPLS model consists of 2 blocks of modeled variation: (1) the Y-predictive $\left(T \mathrm{p} P \mathrm{p}^{\mathrm{T}}\right)$ block, which represents the interclass variation, and (2) the Y-orthogonal $\left(T_{\mathrm{o}} P_{\mathrm{O}}{ }^{\mathrm{T}}\right)$ block, also referred to as the uncorrelated variation, which constitutes the intraclass variation (Wiklund et al., 2008).

The quality of the OPLS-DA model was evaluated by the index of the goodness of fit $\left[\mathbf{R}^{2}(\mathbf{X})\right]$, which displays the fraction of the total variation for the explanatory variables modeled by the selected PLS component; $\mathbf{R}^{2}(\mathbf{Y})$, which displays the fraction of the total variation for the response variable explained by the model; and the index of the prediction power $\left[\mathbf{Q}^{2}(\mathbf{Y})\right]$, which shows the fraction of the total variation of $Y$ that can be predicted by a component, as estimated by crossvalidation. The $\mathrm{Q}^{2}(\mathrm{Y})$ was calculated by 7 -fold crossvalidation based on the default settings of SIMCA-P+ software. The S-plot can visualize the impact of variables on the model. The axes of the S-plot, on data matrix $\mathbf{X}$ and score vector $\mathbf{t}$, represent covariation (magnitude of contribution) in the horizontal axis and correlation (reliability).

The correlations between scores for sensory attributes and measurements by GC-MS were investigated by PLS analysis. The GC-MS peak intensities and the scores for sensory attributes were measured in triplicate. As data pretreatment, Pareto scaling for GC-MS peak intensity data and auto-scaling (transformed so that each variable had a unit variance and zero mean) for sensory evaluation data were used. When the peaks with strong intensity play an important role and the peaks with medium intensity indicate importance as well, Pareto scaling is an effective alternative to metabolomics using mass spectrometry to reduce the risk of intensifying noisy features in the data and give weight to the peaks with medium intensity. Pareto scaling was computed by dividing each peak intensity centered zero-mean by the square root of the standard deviation (Eriksson et al., 2001).

The number of latent factors (PLS components) in the PLS model was determined by computing the optimum number at the balance between $R^{2}$ and $Q^{2}$ with the SIMCA-P software algorithm. After fitting the model, $\mathrm{R}^{2}$ and $\mathrm{Q}^{2}$ were calculated as the index of model quality. In addition, each sample group of different ripening temperatures was used as a prediction set for external model validation. For example, the PLS model was fitted to the samples with ripening temperatures of 10 and $15^{\circ} \mathrm{C}$ used as the calibration set and then samples with a ripening temperature of $5^{\circ} \mathrm{C}$ were used as the prediction set. Model quality was evaluated by 2 parameters, root mean square error of the estimation for observations (RMSEE) for the calibration set and root mean square error of prediction (RMSEP) for the prediction set. The former is the standard deviation of the calibrated residuals and the latter is the predicted residuals (errors). They are computed as the square root of [S (observed value - predicted value $)^{2} / \mathrm{n}$ (number of samples)].

\section{RESULTS AND DISCUSSION}

\section{Metabolic Profiling of 3 Types of Cheddar Cheese}

All samples, which were 3 kinds of Cheddar cheeses $(\mathrm{A}, \mathrm{B}$, and $\mathrm{C})$ of ripening temperatures 5,10 , and $15^{\circ} \mathrm{C}$ for $1,3,6$, and 9 mo, were analyzed using GC-MS, resulting in the annotation of 190 peaks, including amino acids, sugars, organic acids, and inorganic acids, and the identification or partial identification (not as a specific compound, but by category) of 55 compounds. 
The data from these 55 identified and semi-identified peaks were summarized and assessed with PCA. The PCA results (score plot) of these data are shown in Figure 1. The principal components of the total variance that accounted for $72.3 \%$ as the first principal component (PC1) and $17.5 \%$ as the second principal component (PC2) were computed.

The 2-dimensional plot structured by $\mathrm{PC} 1$ and $\mathrm{PC} 2$ displayed from each cheese sample group (A, B, and C) was located according to ripening time (see the 3 arrows in Figure 1a), and even the samples with the same ripening time were plotted in different positions, depending on the ripening temperature. As shown by the arrows in Figure 1a for every sample group of A, $\mathrm{B}$, and $\mathrm{C}$, it was confirmed that the metabolic profile changed as maturation proceeded, which means that the increase or decrease of different compounds varied, depending on manufacturing conditions such as the ingredients used. Additionally, the effect of differences in $\mathrm{NaCl}$ content (A vs. B) or differences in lactic acid bacteria starter (A vs. C) on the difference in metabolic profile was revealed visually by focusing on the closeness and direction of the arrows attached to a particular sample group of $\mathrm{A}, \mathrm{B}$, and $\mathrm{C}$.

A PCA loading plot (Figure 1b) provided the information about the effect of the differences in salt
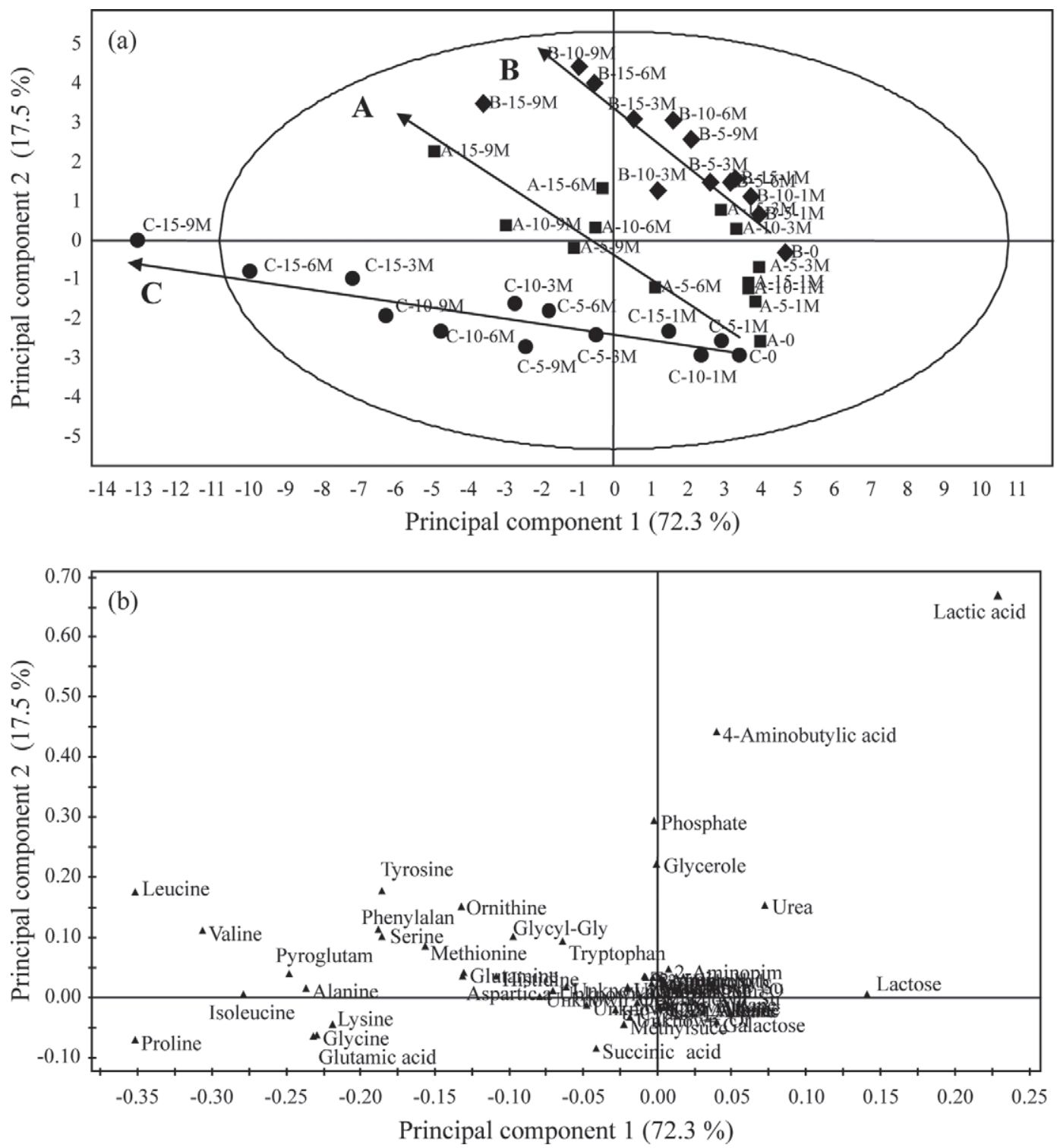

Figure 1. Principal components analysis plots for all samples based on the GC-MS metabolome. (a) Score plots and (b) loading plot for all samples. Cheese A; Cheese B; Cheese C; A-0M, B-0M, and C-0M are prematured samples of cheeses A, B, and C, respectively. For example, A-5-1M means cheese A with ripening conditions of $5^{\circ} \mathrm{C}$ for 1 mo (samples A-0, B-0, and C-0 are samples before ripening). 
content and starter on the changes in the metabolites during ripening. The knowledge on the differences in salt content was obtained from comparison between cheese A and B. Taking a closer look at the time-course sample at each ripening temperature of cheese $\mathrm{A}$, the samples beginning from A-0 (sample before ripening) show a tendency that time-course samples are located along the positive direction of the $\mathrm{PC} 2$ axis initially, and then are located along the negative direction of the PC1 axis. This means that the compounds urea, glycerin, phosphate, 4-aminobutyric acid, and lactic acid increased initially, and then amino acids increased, which seems to be 2-stage transition. On the other hand, the samples of cheese B, beginning from B-0 (sample before ripening), showed a tendency for time-course samples to be located toward the top left in Figure $1 \mathrm{~b}$ (along both the positive direction of the $\mathrm{PC} 2$ axis and the negative direction of the PC1 axis) linearly. This means that the compounds urea, glycerin, phosphate, 4-aminobutyric acid, and amino acids increased simultaneously. The knowledge on the differences in starter was obtained from comparison between cheeses $\mathrm{A}$ and $\mathrm{C}$. The samples of cheese $\mathrm{C}$ beginning from $\mathrm{C}-0$ (sample before ripening) show a tendency that timecourse samples are located along the negative direction of the PC1 axis and not so much along the direction of the PC2 axis, compared with those of cheese A. This means that the amino acids proline, leucine, valine, and isoleucine increased sharply, whereas the compounds urea, glycerin, phosphate, and 4-aminobutyric acid were hardly formed.

As previously mentioned, even the samples with the same ripening time were plotted in different positions of the PCA plot (Figure 1), depending on the ripening temperature. Because the sample $\mathrm{C}$ group displayed especially remarkable distance between mo 0 and 9 in the case of $15^{\circ} \mathrm{C}$, the different kinds of lactic acid bacteria starter could show different sensitivities to ripening temperature.

Additionally, with the application of these PCA plots of the metabolic profiles for time-course samples during ripening under various ripening conditions (for example, if the ripening time is intended to be shortened by setting the ripening temperature higher), the ripening conditions (time and temperature) can be determined to obtain a similar component profile; that is, a close position on the PCA plot. The metabolic profiling technique provides an evidence-based design for cheese ripening conditions.

In the course of cheese product development, if the impact of alteration of the ingredients on the changing of the metabolic profile during the ripening process, and specifically which compounds would increase or decrease, is revealed, this becomes useful information for the design of products and process conditions. Therefore, we identified the significant metabolites that differentiate cheese samples $\mathrm{A}, \mathrm{B}$, and $\mathrm{C}$ by adopting the discriminant analysis with the S-plot of OPLS-DA. A compound with a high absolute value on the horizontal axis $[\operatorname{Cov}(t, X)]$ has a good contribution to discrimination, whereas a compound with a high absolute value on the vertical axis $[\operatorname{Corr}(t, X)]$ has a good reliability with a narrow confidence interval. The vertical axis $\operatorname{Corr}(\mathrm{t}, \mathrm{X})$ ranges from -1 to 1 ; $\operatorname{Corr}(\mathrm{t}, \mathrm{X})>|0.5|$ (Llorach et al., 2010) and $\operatorname{Cov}(t, X)>|0.15|$ were set as tentative thresholds.

First, by comparing cheese A (normal salt type) and cheese B (salt-free type), the effect of salt content on the metabolic profile was investigated with discrimination analysis using OPLS-DA. The model generated one predictive component and one orthogonal component, yielding $\mathrm{R}^{2}(\mathrm{X})=0.921, \mathrm{R}^{2}(\mathrm{Y})=0.727$, and $\mathrm{Q}^{2}(\mathrm{Y})=$ $0.711 ; \mathrm{R}^{2}(\mathrm{X})$ and $\mathrm{R}^{2}(\mathrm{Y})$ are the indices of how well the predictive fraction in the OPLS-DA model account for the $\mathrm{X}$ and the $\mathrm{Y}$ axes, respectively. In this case, $92.1 \%$ of the total variance in the $\mathrm{x}$-axis was explained by the model and this $\mathrm{X}$ variance was used to explain $72.7 \%$ of the total variance in the $y$-axis. In addition, $Q^{2}(Y)$ is the index of predictive ability estimated by cross-validation; that is, the index of the ability to discriminate a new sample as being cheese $A$ or cheese $B ; R^{2}(Y)$ and $\mathrm{Q}^{2}(\mathrm{Y})$ are the indices of model quality of discrimination between cheese A and cheese B. Both $R^{2}(Y)$ and $\mathrm{Q}^{2}(\mathrm{Y})$ were slightly larger than 0.7 , which represented a comparatively good but less than ideal model.

Figure 2A shows the score plot and S-plot of OPLSDA in a comparison between cheeses A and B. In the score plot, the horizontal axis $t_{p}$ represents the predictive score, which is the interclass variation, and the vertical axis $t_{0}$ represents the identical orthogonal score, which is the intraclass variation. The score plot in Figure 2A showed that although cheese A and cheese $\mathrm{B}$ were classified, the variation in the predictive score was conspicuous even within the class; additionally, one sample of cheese A existed in the cheese B zone, which suggested that the difference in metabolic profiles between cheese A and cheese B was not great, as indicated by the approximately 0.7 value of the discriminative indices $\mathrm{R}^{2}(\mathrm{Y})$ and $\mathrm{Q}^{2}(\mathrm{Y})$. Compounds that contribute to class discrimination were determined from the S-plot in Figure 2A. Lactose and galactose were determined as the compounds that were in greater amounts in cheese $\mathrm{A}$ and lesser amounts in cheese B. Lactic acid, 4-aminobutyric acid, and phosphate were determined as the compounds that were in greater amounts in cheese B and lesser amounts in cheese A.

The effect of the kind of lactic acid bacteria starter on metabolic profile was investigated with discrimi- 
(A)
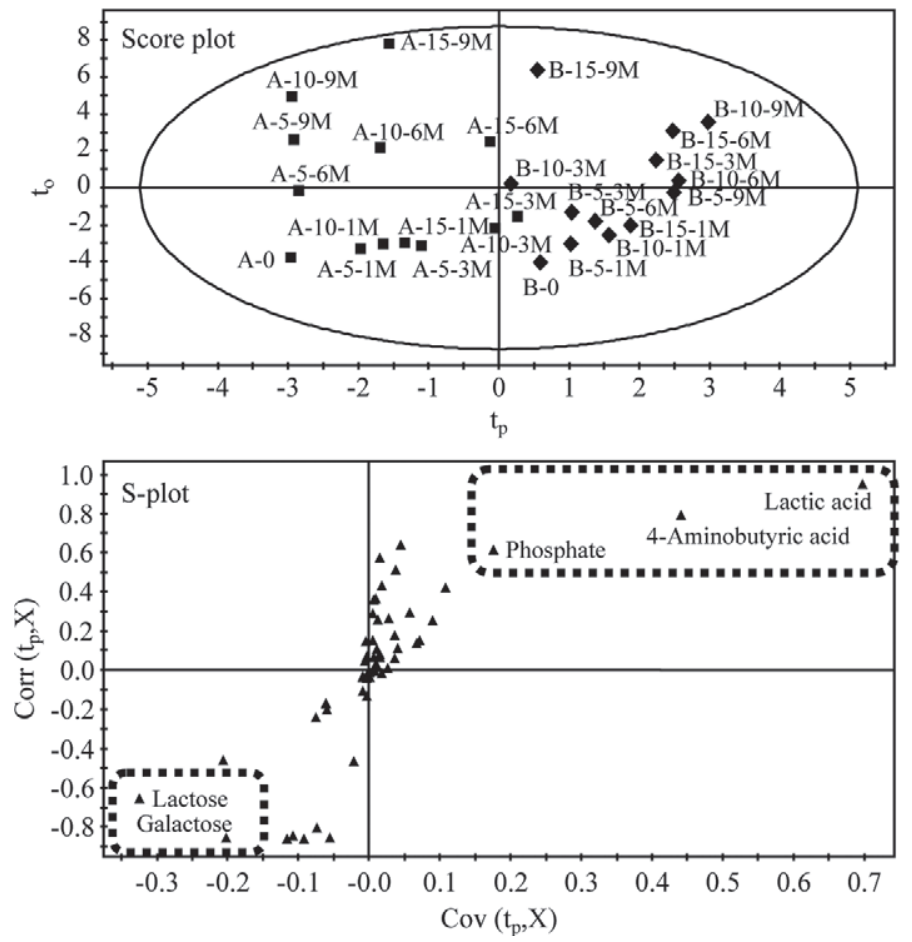

(B)
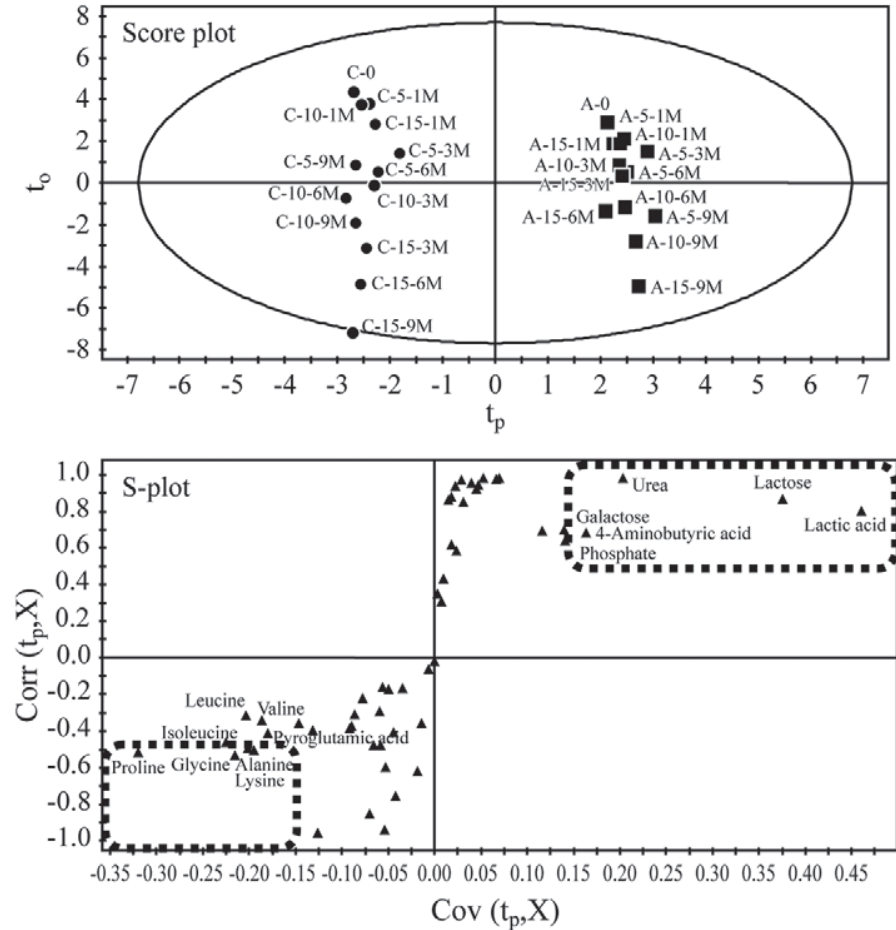

Figure 2. Score plots and S-plots for orthogonal partial least squares-discriminant analysis models. (A) Cheddar cheese sample A versus Cheddar cheese sample B. (B) Cheddar cheese sample A versus Cheddar cheese sample C. A-0M, B-0M, and C-0M are prematured samples of cheeses A, B, and C, respectively. For example, C-5-1M means cheese $\mathrm{C}$ with ripening conditions of $5^{\circ} \mathrm{C}$ for 1 mo (samples A-0, B-0, and C-0 are samples before ripening). The dotted squares in the S-plot are the threshold zone, representing beyond $|0.5|$ in the vertical axis, and beyond $|0.15|$ in the horizontal axis. $t_{o}=$ identical orthogonal score (the intraclass variation); $t_{p}=$ predictive score (the interclass variation); Corr $=$ correlation; $\mathrm{Cov}=$ covariate.

nate analysis using OPLS-DA by comparing cheese A and cheese $\mathrm{C}$, which were both salt-type cheeses, but had different lactic acid bacteria starters. This model generated 1 predictive component and 3 orthogonal components, showing $\mathrm{R}^{2}(\mathrm{X})=0.974, \mathrm{R}^{2}(\mathrm{Y})=0.989$, and $\mathrm{Q}^{2}(\mathrm{Y})=0.977$, which gave excellent model quality. Figure 2B shows the score plot and S-plot of OPLS-DA in the comparison between cheeses $\mathrm{A}$ and $\mathrm{C}$. In the score plot of Figure 2B, distinctive class discrimination was displayed, which showed that the difference of lactic acid bacteria starter caused an obvious difference in metabolic profile. Compounds that contribute to class discrimination between cheese $\mathrm{A}$ and cheese $\mathrm{C}$ were extracted from the S-plot in Figure 2B. Lactic acid, lactose, urea, 4-aminobutyric acid, galactose, and phosphate were determined to be the compounds that were found in greater amounts in cheese A and lesser amounts in cheese C. Proline, glycine, alanine, and lysine existed in the threshold zone (the dotted square in the figure), and isoleucine, leucine, valine, and pyroglutamic acid, representing not beyond $|0.5|$ in the vertical axis, but beyond $|0.15|$ in the horizontal axis, were determined to be the compounds that were found in greater amounts in cheese $\mathrm{C}$ and lesser amounts in cheese A.

Thus, the degree of difference in the metabolic profiles and characteristic compounds contributing to the differences between samples (A vs. B or A vs. C), which used different ingredients, was investigated using OPLS-DA. After some characteristic compounds were identified by the S-plot of OPLS-DA, the extent to which the quantities of these compounds differed depending on the samples was compared by box-andwhisker plots (data not shown). As a result of comparing cheese $\mathrm{A}$ and cheese $\mathrm{C}$, lactic acid, lactose, urea, 4-aminobutyric acid, galactose, and phosphate, which were determined to be the compounds that were found in greater amounts in cheese A and lesser amounts in cheese $\mathrm{C}$, were so characteristic as to be discriminant markers for these 2 sample groups because the distribution of relative peak intensities between cheese A group and cheese $\mathrm{C}$ group during ripening did not overlap, or at least the quartile range did not overlap. On the other hand, for the amino acids proline, glycine, alanine, lysine, isoleucine, leucine, valine, and pyroglutamic acid, which were determined to be the compounds that were 
found in greater amounts in cheese A and lesser amounts in cheese $\mathrm{C}$, although the quartile range of distribution of relative peak intensities during ripening overlapped between cheese A group and cheese C group, a distinctive difference in the median or maximum value was recognized, which meant that these amino acids could be informative as ripening time went by.

The combination of metabolic profiling and OPLSDA quantitatively provided significant information relating to the ripening process; that is to say, which compounds exhibited the characteristic behavior and how their amounts vary during maturation.

\section{Relationship Between Metabolic Profile and Sensory Attributes}

In this report, we described the course of ripening as transitions in the metabolic profile. Additionally, revealing the relationship between the transition in the metabolic profile and the changing of sensory characteristics will allow us to add the sensory evaluation score to the index for the design of ripening conditions and the monitoring of the ripening process. Accordingly, we investigated the relationship between the ripening conditions and sensory scores by conducting sensory predictive modeling using the metabolic profile of ripening time course samples for cheese C.

In our previous reports (Ochi et al., 2012a,b), metabolic profiling using GC-MS and metabolic fingerprinting using a gas chromatography-flame ionization detector targeting hydrophilic low-molecular-weight compounds enabled us to predict precisely 2 sensory attributes, "rich flavor" and "sour flavor," defined by QDA methodology. The characteristics of these sensory attributes were "thick and rich, including umami taste and soy sauce-like flavor" for "rich flavor," and "fundamental taste, typical of lactic and citric acids" for "sour flavor."

The results of the sensory evaluation of cheese C, with a full score on a sensory evaluation of 15 , were as follows. The minimum, maximum, and range (the minimum subtracted from the maximum) values of the sensory score for "rich flavor" were 5.45, 11.22, and 5.76, respectively. For "rich flavor," the sensory score increased with the passage of ripening time. The minimum, maximum, and range values of the sensory score for "sour flavor" were $6.15,8.34$, and 2.19 , respectively, and apart from the sample at $15^{\circ} \mathrm{C}$ for 9 mo, which represented the maximum value, the range was approximately 1.0. Therefore, because the change in sensory score during ripening was relatively slight, "sour flavor" was thought to be lacking in significance for constructing a sensory prediction model in the present study.
One possible reason for the slight change in the sensory score for "sour flavor" during ripening could be the weak formation of metabolites relating to sourness by the lactic acid bacteria starter used for cheese C. The PCA score and loading plot in Figure 1 show that samples of cheese $\mathrm{C}$ did not have a large distribution along the positive direction of $\mathrm{PC} 2$ and $\mathrm{PC} 2$ had a high correlation to the sour taste compounds, judging from the high loading values of lactic acid, 4-aminobutyric acid, and phosphate for $\mathrm{PC} 2$. These results fit in with the inference mentioned above. In this report, therefore, we modeled the relationship between metabolic profile and "rich flavor" score, which increased over the course of ripening time, using PLS.

Analysis of variance showed that the sensory attribute "rich flavor" had highly significant differences $(P$ $<0.001$ ) among cheese $\mathrm{C}$ samples during ripening, and the result of the Tukey honestly significant difference multiple comparison test identified where significant differences existed. Samples of cheese $\mathrm{C}$ that did not have significant differences in the values of least squares means were put in parentheses, and then 8 groups were developed as follows: $\left(15^{\circ} \mathrm{C}-9 \mathrm{M}, 15^{\circ} \mathrm{C}-6 \mathrm{M}\right),\left(15^{\circ} \mathrm{C}-6 \mathrm{M}\right.$, $\left.10^{\circ} \mathrm{C}-9 \mathrm{M}, 10^{\circ} \mathrm{C}-6 \mathrm{M}\right),\left(10^{\circ} \mathrm{C}-9 \mathrm{M}, 10^{\circ} \mathrm{C}-6 \mathrm{M}, 15^{\circ} \mathrm{C}-3 \mathrm{M}\right)$, $\left(15^{\circ} \mathrm{C}-3 \mathrm{M}, \quad 5^{\circ} \mathrm{C}-9 \mathrm{M}, \quad 10^{\circ} \mathrm{C}-3 \mathrm{M}\right), \quad\left(5^{\circ} \mathrm{C}-9 \mathrm{M}, \quad 10^{\circ} \mathrm{C}-3 \mathrm{M}\right.$, $\left.15^{\circ} \mathrm{C}-1 \mathrm{M}, 5^{\circ} \mathrm{C}-3 \mathrm{M}, 5^{\circ} \mathrm{C}-6 \mathrm{M}\right),\left(10^{\circ} \mathrm{C}-3 \mathrm{M}, 15^{\circ} \mathrm{C}-1 \mathrm{M}, 5^{\circ} \mathrm{C}-\right.$ $\left.3 \mathrm{M}, 5^{\circ} \mathrm{C}-6 \mathrm{M}, 10^{\circ} \mathrm{C}-1 \mathrm{M}\right),\left(15^{\circ} \mathrm{C}-1 \mathrm{M}, 5^{\circ} \mathrm{C}-3 \mathrm{M}, 5^{\circ} \mathrm{C}-6 \mathrm{M}\right.$, $\left.10^{\circ} \mathrm{C}-1 \mathrm{M}, \quad 5^{\circ} \mathrm{C}-1 \mathrm{M}\right)$, and $\left(10^{\circ} \mathrm{C}-1 \mathrm{M}, \quad 5^{\circ} \mathrm{C}-1 \mathrm{M}, \quad-0 \mathrm{M}\right)$, where the temperature is the ripening temperature and $\mathrm{M}$ represents the months of ripening.

In the PLS regression analysis, the sensory score for the sensory attribute "rich flavor" was the $\mathrm{Y}$ variable (object variable) and the peak intensity data from metabolome analysis were the $\mathrm{X}$ variables (explanatory variables). The sample group at each ripening temperature was used as a prediction set to validate the prediction capacity of the PLS model. First, the PLS model was fitted to the samples at ripening temperatures of 10 and $15^{\circ} \mathrm{C}$ used as the calibration set, and then validation with samples at the ripening temperature of $5^{\circ} \mathrm{C}$ used as the prediction set was carried out. This procedure was conducted for all ripening temperatures. The results of validation, namely the average values of the prediction index for "rich flavor," were the number of PLS components $=1.67, \mathrm{R}^{2}=0.964, \mathrm{Q}^{2}=0.943$, RMSEE $=0.360$, and RMSEP $=0.616$, which showed that a model with satisfactory fitting and predictability was obtained.

Additionally, all samples of cheese $\mathrm{C}$ during ripening were used to construct the PLS model to extract information about the compounds contributing to the sensory prediction model for "rich flavor." The predicted versus measured figures, representing the relationship between the predicted sensory score from metabolome 
analysis and the measured sensory score by the panel of "rich flavor," are shown in Figure 3. This model exhibited the number of PLS components $=2, \mathrm{R}^{2}=0.959$, $\mathrm{Q}^{2}=0.929$, and RMSEE $=0.374$, which showed an excellent fit. Important metabolites for constructing the sensory prediction model were identified from variable importance for the projection (VIP) values. Variable importance for the projection is an index computed from the influence of every X-term (metabolite) on a Y-variable (sensory attribute) in the PLS model and the VIP of one term can be compared with the others. The X-terms with large VIP values (greater than 1) are the most relevant for explaining Y (Eriksson et al., 2001). The compounds contributing significantly [i.e., large VIP values $(>1)$ ] to the prediction model for "rich flavor" are shown in Table 1. Many amino acids and lactic acid were listed as the compounds contributing to the model construction. These are proline, leucine, valine, isoleucine, pyroglutamic acid, alanine, glutamic acid, glycine, lysine, lactic acid, tyrosine, serine, phenylalanine, methionine, aspartic acid, and ornithine, in descending order of contribution. These compounds can be marker candidates for ripening, which will be discussed in the next section.

Regarding the sensory predictive model for "rich flavor," although the constructed model in the present study is applied to quite different samples from those of the constructed model in the previous study (Ochi et al., 2012a,b), both models exhibit excellent fitting and predictability. This suggests that our analytical method focusing on hydrophilic low-molecular-weight components is relevant to represent the sensory attribute of
Table 1. List of important compounds that influence variables used to create the partial least squares regression model for "rich flavor"

\begin{tabular}{ll}
\hline Compound & VIP $^{1}$ \\
\hline Proline & 2.613 \\
Leucine & 2.463 \\
Valine & 2.245 \\
Isoleucine & 2.105 \\
Pyroglutamic acid & 1.860 \\
Alanine & 1.728 \\
Glutamic acid & 1.634 \\
Glycine & 1.632 \\
Lysine & 1.615 \\
Lactic acid & 1.540 \\
Tyrosine & 1.530 \\
Serine & 1.437 \\
Phenylalanine & 1.434 \\
Methionine & 1.156 \\
Aspartic acid & 1.042 \\
Ornithine & 1.007
\end{tabular}

${ }^{1}$ Variable importance for the projection, which summarizes the importance of the variables both to explain $\mathrm{X}$ and to correlate to $\mathrm{Y}$ in the partial least squares regression model.

"rich flavor." On the other hand, the kind and order of metabolites as contributors for model construction and this variance would provide important knowledge on the samples at the compound level.

In the case of metabolome changing with ripening, we accomplished the modeling of the relationship between metabolic profile and sensory attribute. This result allows us to determine the ripening conditions (temperature and time) to obtain any sensory score for "rich flavor," which is thought to be a significant sensory attribute for matured-type cheeses, at the ripening condition design stage. Additionally,

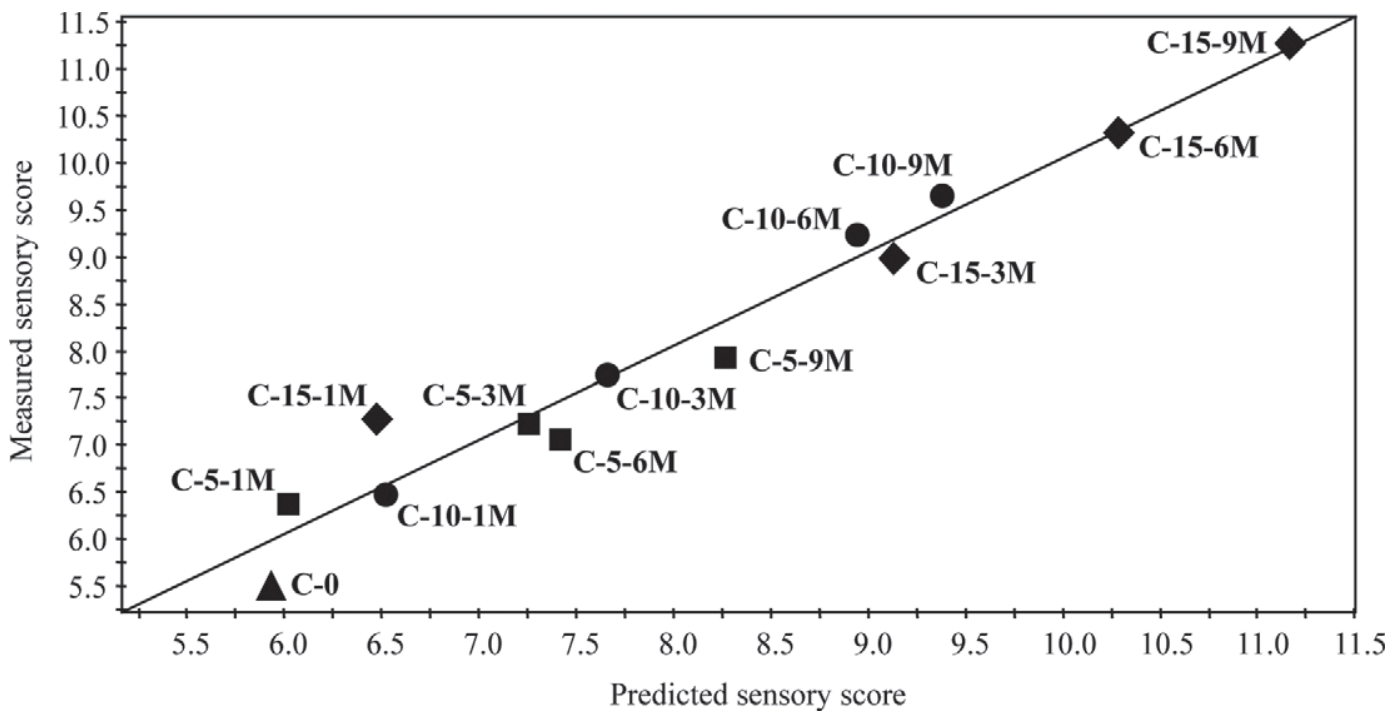

Figure 3. Relationships between predicted and measured quantitative descriptive sensory analysis (QDA) scores for "rich flavor" based on the partial least squares regression model for cheese $\mathrm{C}$ samples. C-0M are prematured samples of cheese C. For example, C-5-1M means cheese $\mathrm{C}$ with ripening conditions of $5^{\circ} \mathrm{C}$ for $1 \mathrm{mo}$ ( $\mathrm{C}-0$ is a sample before ripening). 
(a)

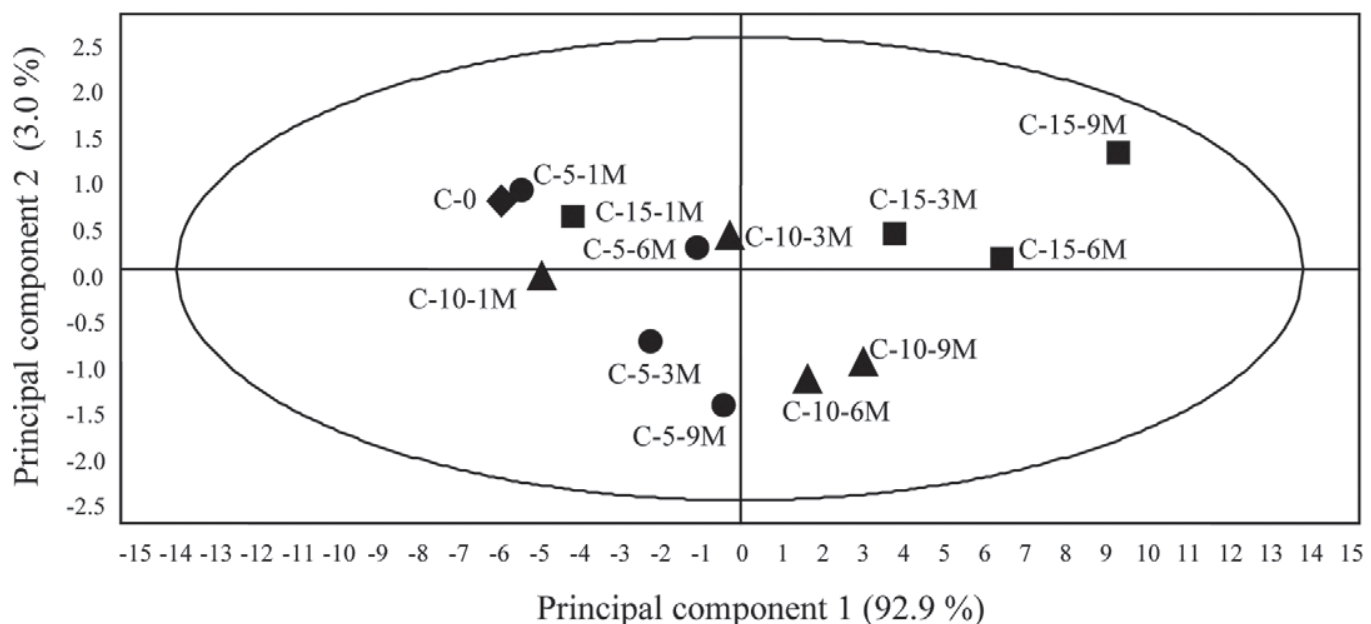

(b)

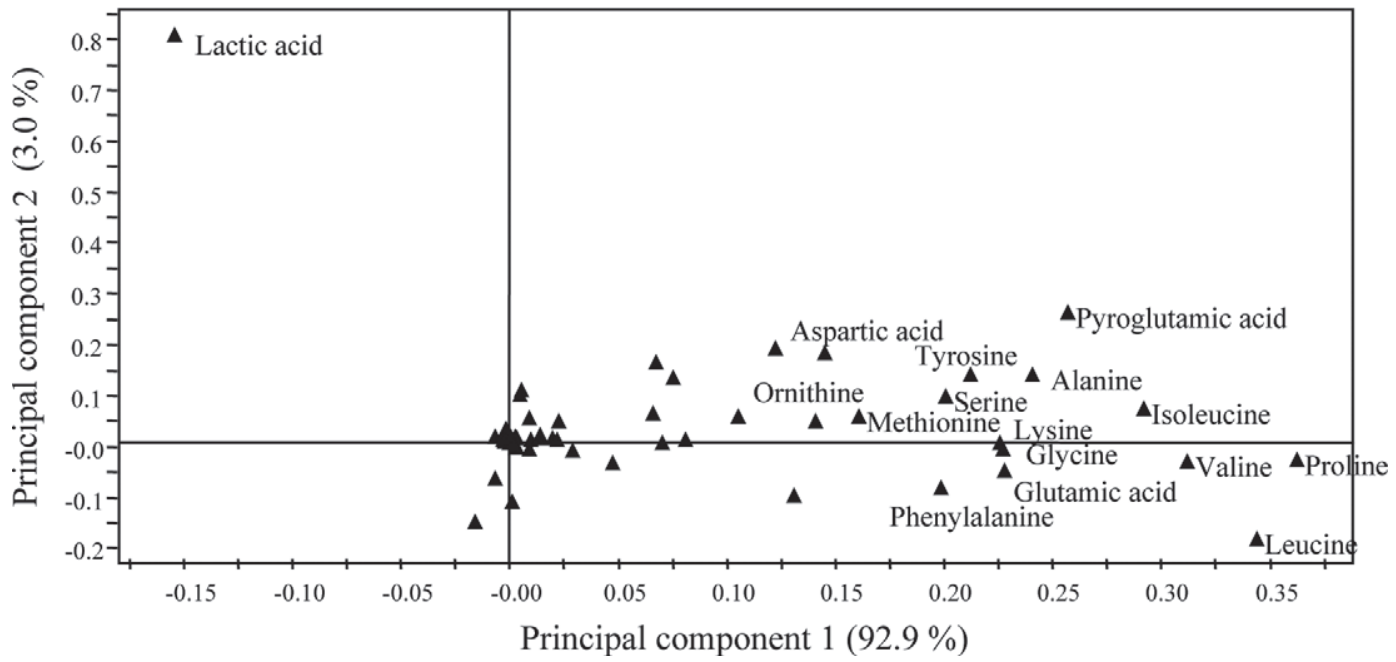

Figure 4. Principal components analysis plots based on the GC-MS metabolome. (a) Score plot and (b) loading plot for Cheddar cheese sample C. C-0M is a prematured sample of cheese C. For example, C-5-1M means cheese $\mathrm{C}$ with ripening conditions of $5{ }^{\circ} \mathrm{C}$ for 1 mo $(\mathrm{C}-0$ is a sample before ripening).

when the monitoring of the cheese ripening process is conducted using this model, cheese quality can be checked in terms of both metabolic profile and sensory score by predicting the intensity of the "rich flavor" from the metabolic profile, in the quality inspection during the manufacturing process and of the finished products.

\section{Selection of Marker Compounds for Monitoring the Cheese Ripening Process}

The PCA for metabolome analysis data on cheese C in the ripening process was performed for the purpose of selecting marker compounds for monitoring cheese ripening. The score plot and loading plot are shown in Figure 4. The principal components that accounted for $92.3 \%$ as $\mathrm{PC} 1$ and $3.0 \%$ as $\mathrm{PC} 2$ of the total variance were computed. The total of these $2 \mathrm{PC}$ summarized approximately $96 \%$ of the total information and PC1 in particular could express most of the variance.

The PCA score plot is shown in Figure 4a, which shows that samples were located along the $\mathrm{PC} 1$ axis from the direction of negative to positive, depending on the ripening time. The PCA loading plot is shown in Figure $4 \mathrm{~b}$, which shows that the amino acids proline, leucine, valine, isoleucine, pyroglutamic acid, alanine, glutamic acid, glycine, lysine, tyrosine, serine, phenylalanine, methionine, aspartic acid, and ornithine were 
identified as compounds contributing to the direction of the positive value on the PC1 axis.

On the other hand, lactic acid was identified as contributing to the direction of the negative value on the PC1 axis. Because the proportion of variance in the PC1 was approximately 93\%, on checking the score plot against the loading plot in Figure 4, large amounts of the amino acids identified by the loading plot were considered to form in the ripening process.

We displayed the compounds contributing significantly to the PLS sensory predictive model for "rich flavor" in Table 1, with the VIP value as an index. These compounds were consistent with the amino acids extracted from the PCA loading plot in Figure 4b in terms of kind and order of contribution. These amino acids and lactic acid were suggested to be strong marker candidates for monitoring the ripening process of cheese C. The transitions of peak intensities of these marker candidate compounds during the ripening period under various conditions are shown in Figure 5. Amino acids, starting with proline, showed that peak intensities represented by the vertical axis increased as ripening time passed and the graphical configurations among these amino acids are similar. Additionally, the higher the ripening temperature was set, the greater the peak intensity was, even in the same ripening period, for these amino acids. Conversely, lactic acid showed large peak intensity, but the change depending on ripening time and ripening temperature was not so remarkable. From these results, the amino acids proline, leucine, valine, isoleucine, pyroglutamic acid, alanine, glutamic acid, glycine, lysine, tyrosine, serine, phenylalanine, methionine, aspartic acid, and ornithine can be relevant markers for monitoring the ripening process.

These amino acids represent the time-dependent increasing behavior during ripening. This increase means the balance between the increase in free amino acids by proteolysis and the decrease in amino acids by catabolism. Proteolysis in cheese during ripening is generally caused by proteases from various sources, including milk, coagulant (e.g., chymosin), starter lactic acid bacteria, and exogenous proteolytic enzymes. The production of amino acids in the present study can be attributed to peptidases in the starter_culture. Various amino acids were liberated during the ripening of cheese $\mathrm{C}$, which showed that aminopeptidase with broad specificity, dipeptidase, and tripeptidase from the starter culture used for this cheese worked actively. Additionally, as a noteworthy result, proline was the top contributor (highest VIP value). Because proline has a unique cyclic structure, specialized peptidases are required (Cunningham and O'Connor, 1997). In lactic acid bacteria, the following proline-specific peptidases have been reported (Tan et al., 1993; Cunningham and
O'Connor, 1997): X-prolyl dipeptidyl aminopeptidase (hydrolyzing specifically X-Pro-Y- . . . and cleaving the N-terminal X-Pro), proline iminopeptidase (releasing an N-terminal proline residue from peptides of any length), and prolidase (hydrolyzing dipeptides containing a C-terminal proline). As for amino acid catabolism, free amino acids in the cheese matrix are taken by lactic acid bacteria, transported to an intracellular amino acid pool, and converted into various flavor compounds through $\alpha$-keto acids, or amines (van Kranenburg et al., 2002). For example, valine, leucine, isoleucine, methionine, cysteine, phenylalanine, tyrosine, and tryptophan were listed as precursors of cheese flavor formed by the catabolism of lactic acid bacteria isolated from Cheddar cheese (Singh et al., 2003). Generated free amino acids and compounds arising from the catabolism of amino acids during the cheese ripening process contribute directly to cheese flavor. It has been suggested that exopeptidase that liberates hydrophobic amino acid can reduce the bitterness of protein hydrolysate. For example, it has been reported that a proline-rich, bitter peptide found in cheese could be degraded by an aminopeptidase produced by a starter culture (Nissen, 1986). In the present study, the relative peak intensities of amino acids considered as relevant to ripening markers for cheese $\mathrm{C}$, displayed by the vertical axis in Figure 5, became greater as ripening time passed under the same ripening temperature and at higher ripening temperature under the same ripening time. This would mean that free amino acids were increased as ripening proceeded and the following flavor development arose by amino acids themselves and volatile compounds, such as amino acid catabolites, which reduced the bitterness in the cheese with resulting strong taste and aroma. The time course increase in the score for "rich flavor" in Figure 3 could support this discussion.

\section{CONCLUSIONS}

We proposed an application methodology that combines metabolic profiling with multiple appropriate multivariate analyses and verified it on the industrial scale for the ripening process of Cheddar cheese to make practical use of profiling hydrophilic low-molecular-weight compounds using GC-MS to design optimal conditions and monitor the quality of the cheese ripening process. The PCA provided an overview of the effect of $\mathrm{NaCl}$ content and the kind of lactic acid bacteria starter on the metabolic profile in the ripening process of Cheddar cheese and the OPLS-DA unveiled the differences in characteristic metabolites. In a comparison between cheese A (normal salt type) and cheese B (saltfree type), using the same lactic acid bacteria starter, the compounds that contributed to class discrimination 
Proline

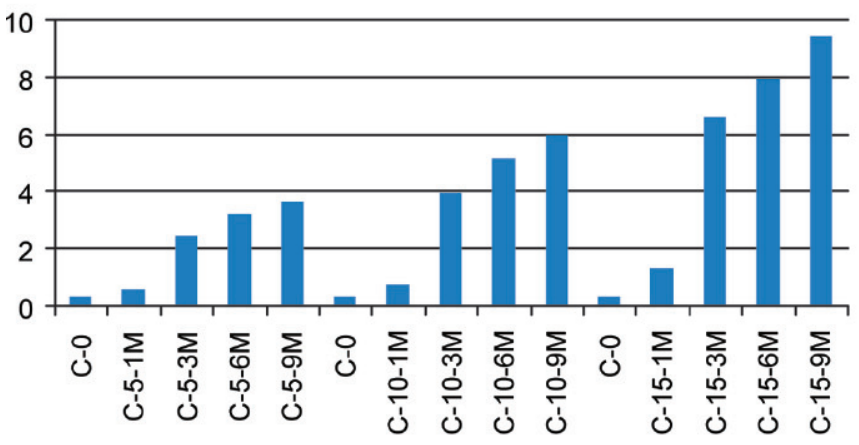

Valine

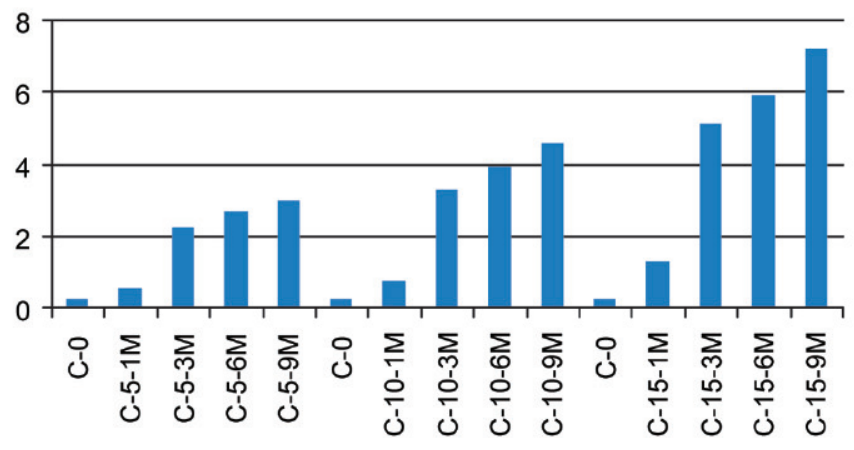

Pyroglutamic acid

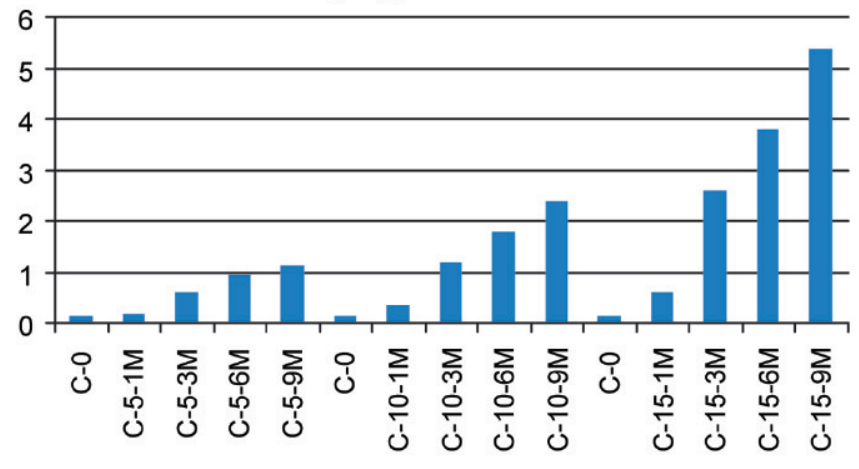

Glutamic acid

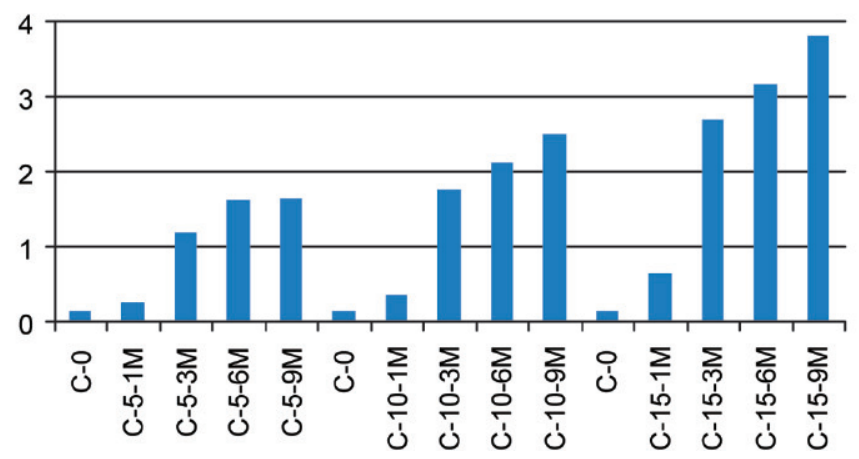

Leucine

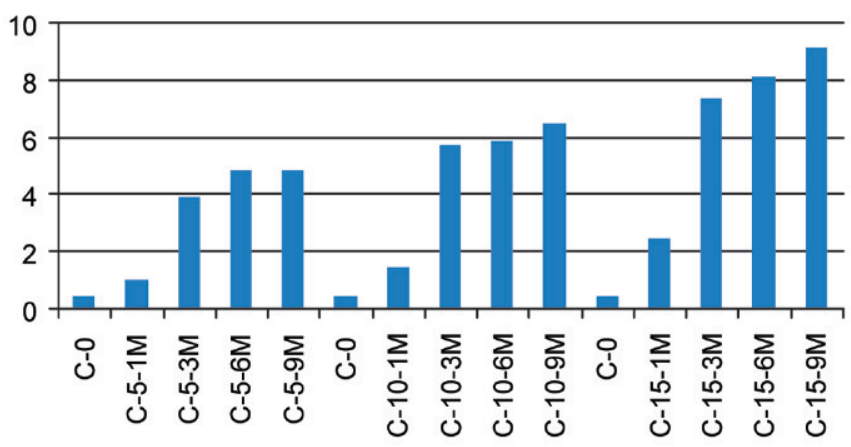

Isoleucine

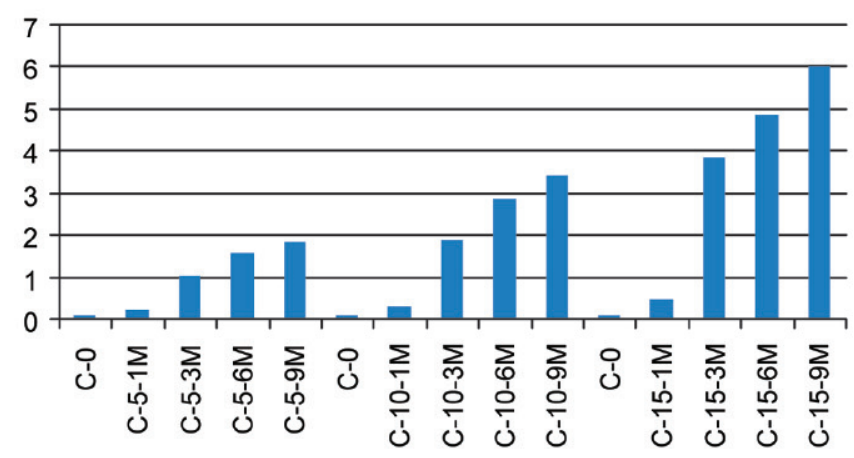

Alanine

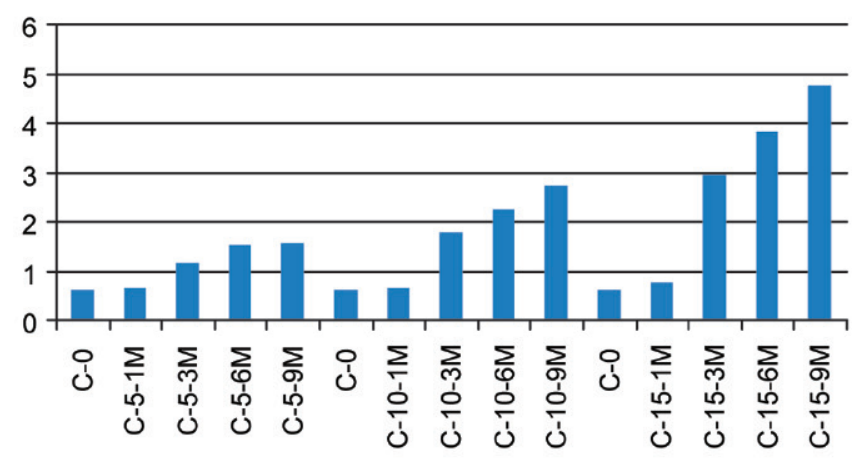

Glycine

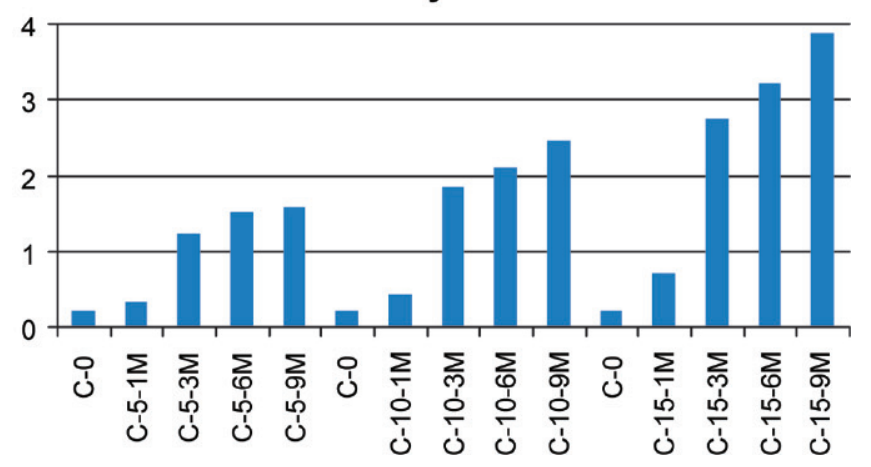

Figure 5. Changes in the peak intensities of marker candidates in the ripening process. For example, $\mathrm{C}-5-1 \mathrm{M}$ means cheese $\mathrm{C}$ with ripening conditions of $5^{\circ} \mathrm{C}$ for $1 \mathrm{mo}$ (C-0 is a sample before ripening). The vertical axis represents the relative peak intensity normalized to the ribitol peak included as the internal standard. Color version available in the online PDF. 
Lysine

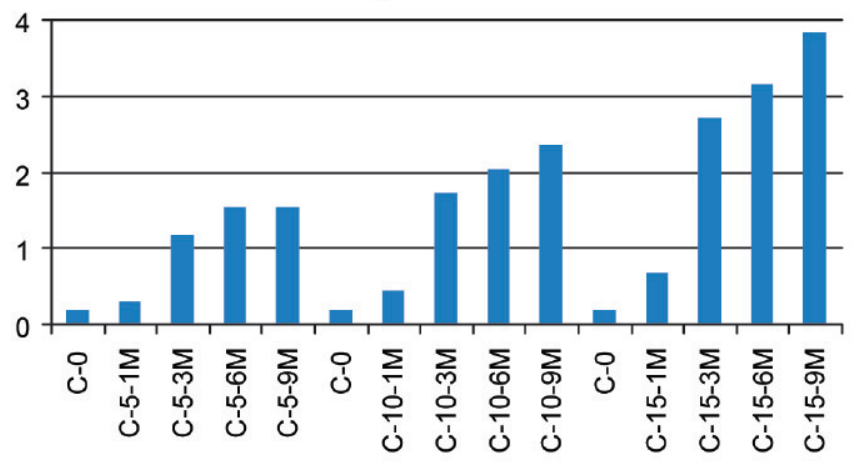

Serine

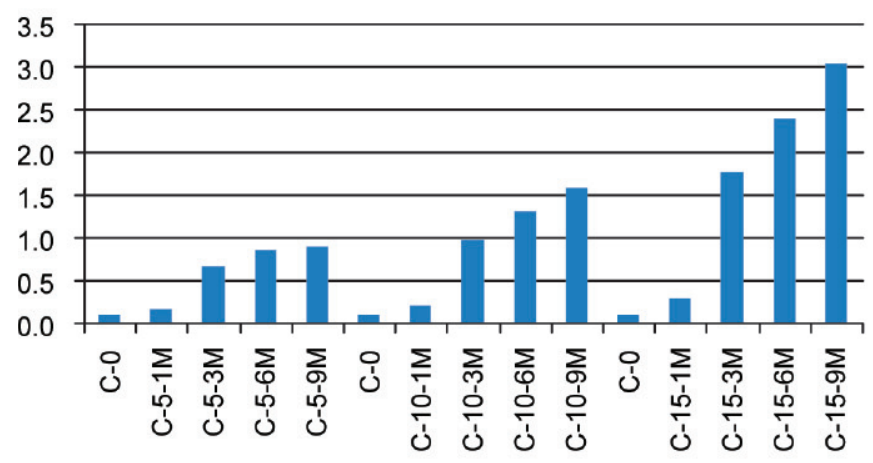

Methionine

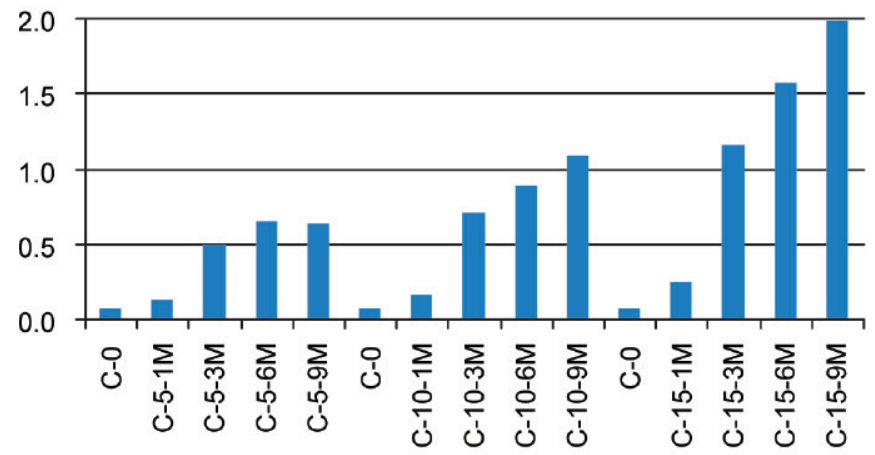

Ornithine

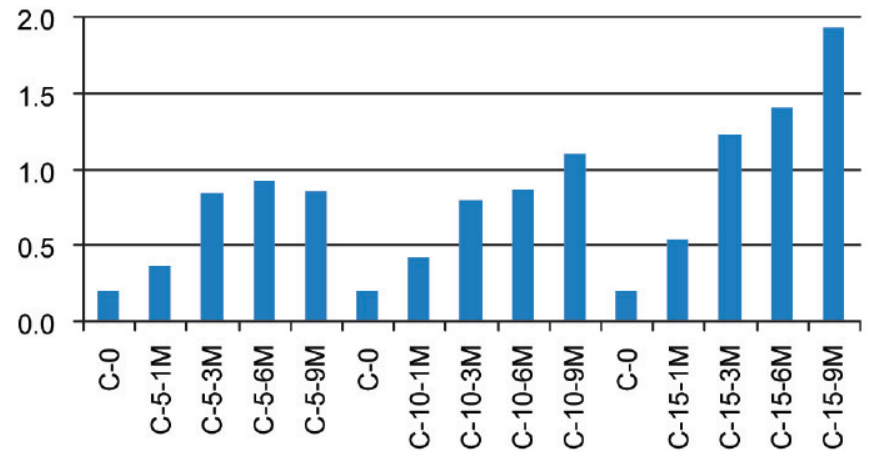

Tyrosine

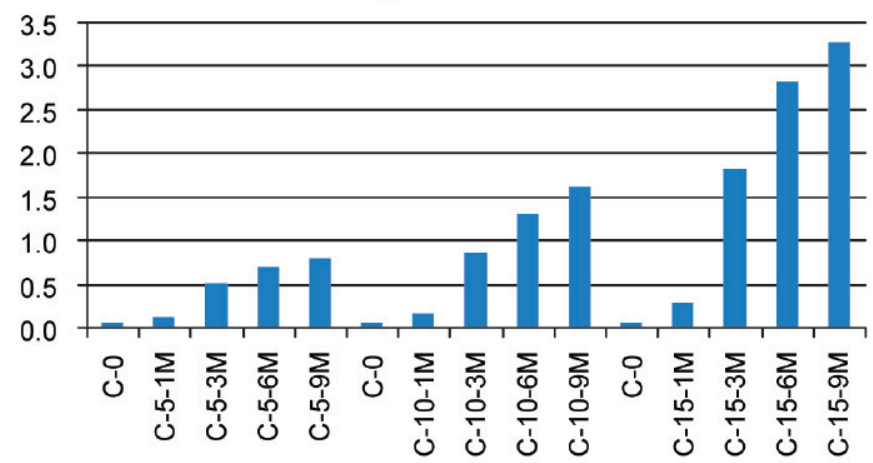

Phenylalanine

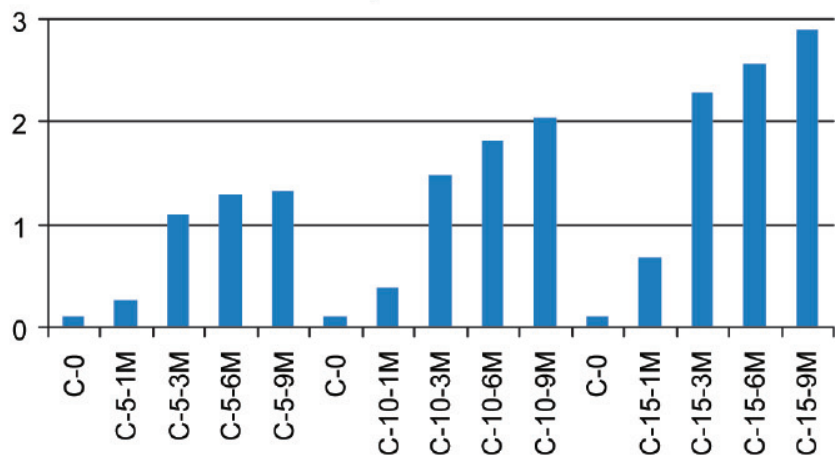

Aspartic acid

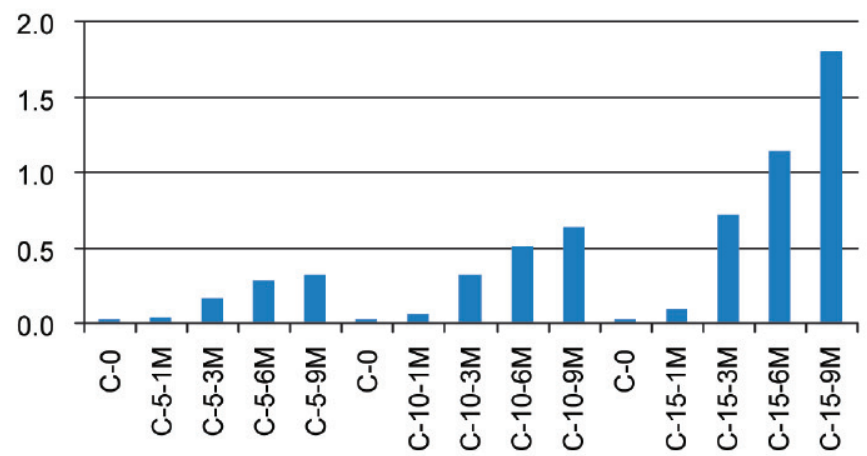

Lactic acid

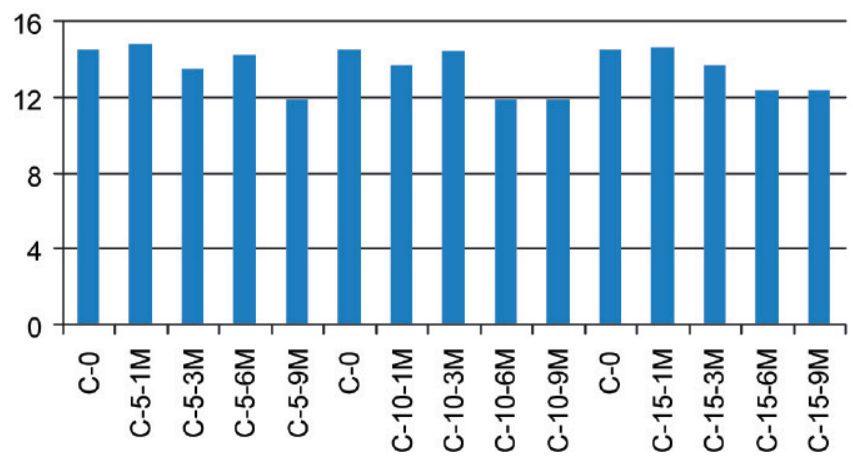

Figure 5 (Continued). Changes in the peak intensities of marker candidates in the ripening process. For example, C-5-1M means cheese C with ripening conditions of $5^{\circ} \mathrm{C}$ for $1 \mathrm{mo}(\mathrm{C}-0$ is a sample before ripening). The vertical axis represents the relative peak intensity normalized to the ribitol peak included as the internal standard. Color version available in the online PDF. 
from the S-plot of OPLS-DA were lactose and galactose as the compounds that existed in greater amounts in cheese A and lesser amounts in cheese B, whereas lactic acid, 4-aminobutyric acid, and phosphate were identified as the compounds that existed in greater amounts in cheese B and lesser amounts in cheese A. On the other hand, in a comparison between cheese A and cheese $\mathrm{C}$, which contained normal salt, but had different lactic acid bacteria starters, the extracted compounds that contributed to class discrimination were lactic acid, lactose, urea, 4-aminobutyric acid, galactose, and phosphate as the compounds that existed in greater amounts in cheese $\mathrm{A}$ and lesser amounts in cheese $\mathrm{C}$, whereas proline, glycine, alanine, lysine, isoleucine, leucine, valine, and pyroglutamic acid were identified as the compounds that existed in greater amounts in cheese $\mathrm{C}$ and lesser amounts in cheese A. A sensory prediction model was then constructed using PLS regression analysis to correlate changes in the metabolic profile during the ripening process as time passed with sensory attributes, resulting in a good predictive model for "rich flavor." The predicted sensory score from this model suggests facts on which to base a judgment in the design of optimal conditions and the monitoring of quality in the ripening process. For example, regarding the monitoring of products in progress or final products in the ripening process, if an extraordinary state occurred, it could be detected by checking the sensory score predicted by metabolomic analysis against the sensory score measured in an actual inspection. Namely, the predicted sensory score was taken into account for condition design and quality monitoring as a qualitative index. Additionally, compounds that can be marker candidates in the ripening process monitoring were identified by the PCA loading plot and the VIP value of the PLS sensory prediction model. In the case of cheese $\mathrm{C}$ in this report, 15 amino acids were identified. The present study provides the technical platform for influencing the ripening process of cheese by the practical implementation of metabolic profiling that targets hydrophilic low-molecular-weight components using GC-MS.

\section{REFERENCES}

Aiello, D., D. De Luca, E. Gionfriddo, A. Naccarato, A. Napoli, E. Romano, A. Russo, G. Sindona, and A. Tagarelli. 2011. Review: Multistage mass spectrometry in quality, safety and origin of foods. Eur. J. Mass Spectrom. (Chichester, Eng.) 17:1-31.

Andersen, L. T., Y. Ardö, and L. P. Bredie. 2010. Study of taste-active compounds in the water-soluble extract of mature Cheddar cheese. Int. Dairy J. 20:528-536.

Biede, S. L., and E. G. Hammond. 1979a. Swiss cheese flavor: I. Chemical analysis. J. Dairy Sci. 62:227-237.

Biede, S. L., and E. G. Hammond. 1979b. Swiss cheese flavor: II. Organoleptic analysis. J. Dairy Sci. 62:238-248.
Cevallos-Cevallos, J. M., and J. I. Reyes-De-Corcuera. 2012. Metabolomics in food science. Adv. Food Nutr. Res. 67:1-24.

Cevallos-Cevallos, J. M., J. I. Reyes-De-Corcuera, E. Etxeberria, M. D. Danyluk, and G. E. Rodrick. 2009. Metabolomic analysis in food science: A review. Trends Food Sci. Technol. 20:557-566.

Cunningham, D. F., and B. O'Connor. 1997. Proline specific peptidases. Biochim. Biophys. Acta 1343:160-186.

Drexler, D. M., M. D. Reily, and P. A. Shipkova. 2011. Advances in mass spectrometry applied to pharmaceutical metabolomics. Anal. Bioanal. Chem. 399:2645-2653.

Engel, E., S. Nicklaus, A. Garem, C. Septier, C. Salles, and J. L. Le Quéré. 2000. Taste active compounds in a goat cheese watersoluble extract. 1. Development and sensory validation of a model water-soluble extract. J. Agric. Food Chem. 48:4252-4259.

Eriksson, L., E. Johansson, N. Kettaneh-Wold, J. Trygg, C. Wikström, and S. Wold. 2001. Multi- and Megavariate Data Analysis. Part II: Advanced Applications and Method Extensions. 2nd revised and enlarged ed. Umetrics AB, Umeå, Sweden.

Herrero, M., C. Simó, V. García-Cañas, E. Ibáñez, and A. Cifuentes. 2012. Foodomics: MS-based strategies in modern food science and nutrition. Mass Spectrom. Rev. 31:49-69.

Kuang, H., Z. Li, C. Peng, L. Liu, L. Xu, Y. Zhu, L. Wang, and C. Xu. 2012. Metabonomics approaches and the potential application in food safety evaluation. Crit. Rev. Food Sci. Nutr. 52:761-774.

Llorach, R., I. Garrido, M. Monagas, M. Urpi-Sarda, S. Tulipani, B. Bartolome, and C. Andres-Lacueva. 2010. Metabolomics study of human urinary metabolome modifications after intake of almond (Prunus dulcis (Mill.) D. A. Webb) skin polyphenols. J. Proteome Res. 9:5859-5867.

Lommen, A. 2009. MetAlign: Interface-driven, versatile metabolomics tool for hyphenated full-scan mass spectrometry data preprocessing. Anal. Chem. 81:3079-3086.

Ma, Y., P. Zhang, Y. Yang, F. Wang, and H. Qin. 2012. Metabolomics in the fields of oncology: A review of recent research. Mol. Biol. Rep. 39:7505-7511.

MacFie, H. J., N. Bratchell, K. Greenhoff, and L. V. Vallis. 1989. Designs to balance the effect of order of presentation and first-order carry-over effects in hall tests. J. Sens. Stud. 4:129-148.

Mannina, L., A. P. Sobolev, and D. Capitani. 2012. Applications of NMR metabolomics to the study of foodstuffs: Truffle, kiwifruit, lettuce, and sea bass. Electrophoresis 33:2290-2313.

McSweeney, P. L. H. 2004. Biochemistry of cheese ripening: Introduction and overview. Pages 347-360 in Cheese: Chemistry, Physics, and Microbiology. Vol. 1. 3rd ed. P. F. Fox, P. L. H. McSweeney, T. M. Cogan, and T. P. Guinee, ed. Elsevier, Amsterdam, the Netherlands.

Meilgaard, M., G. V. Civille, and B. T. Car. 2006. Triangle test. Pages 65-72 in Sensory Evaluation Techniques. 4th ed. CRC Press Inc., Boca Raton, FL.

Nadella, K. D., S. S. Marla, and P. A. Kumar. 2012. Metabolomics in agriculture. OMICS 16:149-159.

Nissen, J. A. 1986. A review of food protein hydrolysis - Specific areas. Pages 57-107 in Enzymic Hydrolysis of Food Proteins, Elsevier Applied Science Publishers Ltd., UK.

Ochi, H., T. Bamba, H. Naito, K. Iwatsuki, and E. Fukusaki. 2012a. Metabolic fingerprinting of hard and semi-hard natural cheeses using GC/FID for practical sensory prediction modeling. J. Biosci. Bioeng. 114:506-511.

Ochi, H., H. Naito, K. Iwatsuki, T. Bamba, and E. Fukusaki. 2012b. Metabolomics-based component profiling of hard and semi-hard natural cheeses with gas chromatography/time-of-flight mass spectrometry, and its application to sensory predictive modeling. J. Biosci. Bioeng. 113:751-758.

Putri, S. P., Y. Nakayama, F. Matsuda, T. Uchikata, S. Kobayashi, A. Matsubara, and E. Fukusaki. 2013. Current metabolomics: Practical applications. J. Biosci. Bioeng. 115:579-589.

Salles, C., C. Septier, F. Roudot-Algaron, A. Guillot, and P. X. Etievant. 1995. Sensory and chemical analysis of fractions obtained by gel permeation of water-soluble Comte cheese extracts. J. Agric. Food Chem. 43:1659-1668. 
Scott, R. 1998 Cheesemaking Practice. 3rd ed. Aspen Publishers Inc., Maryland, UK.

Singh, T. K., M. A. Drake, and K. R. Cadwallader. 2003. Flavor of Cheddar cheese: A chemical and sensory perspective. Compr. Rev. Food Sci. Food Saf. 2:139-162.

Stone, H., and J. L. Sidel. 2004. Descriptive analysis. Pages 201-244 in Sensory Evaluation Practices. 3rd ed. Elsevier Academic Press, San Diego, CA.

Tan, P. S. T., B. Poolman, and W. N. Konings. 1993. Proteolytic enzymes of Lactococcus lactis. J. Dairy Res. 60:269-286.

Toelstede, S., and T. Hofmann. 2008. Quantitative studies and taste re-engineering experiments toward the decoding of the nonvolatile sensometabolome of Gouda cheese. J. Agric. Food Chem. 56:5299-5307.

Tsugawa, H., T. Bamba, M. Shinohara, S. Nishiumi, M. Yoshida, and E. Fukusaki. 2011. Practical non-targeted gas chromatography/ mass spectrometry-based metabolomics platform for metabolic phenotype analysis. J. Biosci. Bioeng. 112:292-298.

van Kranenburg, R. V., M. Kleerebezem, J. van Hylckama Vlieg, B. M. Ursing, J. Boekhorst, B. A. Smit, E. H. E. Ayad, G. Smit, and R. J. Siezen. 2002. Flavour formation from amino acids by lactic acid bacteria: Predictions from genome sequence analysis. Int. Dairy J. 12:111-121.

Wiklund, S., E. Johansson, L. Sjöström, E. J. Mellerowicz, U. Edlund, J. P. Shockcor, J. Gottfries, T. Moritz, and J. Trygg. 2008. Visualization of GC/TOF-MS-based metabolomics data for identification of biochemically interesting compounds using OPLS class models. Anal. Chem. 80:115-122.

Wishart, D. S. 2008. Applications of metabolomics in drug discovery and development. Drugs R D. 9:307-322. 